\title{
INVESTIGATION OF ENERGY LEVELS AND ELECTROMAGNETIC TRANSITIONS FOR Yb-Pt NUCLEI WITH $N=108$ USING IBM, IVBM, AND BMM
}

\begin{abstract}
The interacting boson and vector boson models, as well as the Bohr-Mottelson one, are employed to describe the energy levels and electromagnetic transitions of the ${ }^{178} \mathrm{Yb}^{186} \mathrm{Pt}$ $(N=108)$ nuclei. For the purpose of determining the evolution of the ground state, both $r((I+2) / I)$ and $E-G O S$ ratios have been calculated as functions of the spin $I$. Based on the interacting vector boson model and Bohr-Mottelson model, the negative-parity and GSB bands have been calculated, while the interacting boson model is only employed to calculate GSB, $\gamma$, and $\beta$. The interacting boson model is also used to calculate the reduced transition probabilities $B(E 2)$. The obtained findings show a very well agreement with experimentally obtained results elsewhere. We also used the intrinsic coherent state to obtain the potential energy surfaces. These results indicate that these nuclei have a rotational property $S U(3)$, while ${ }^{186} P t$ has property $O(6)$.

Ke ywords: IBM, IVBM, BMM, energy level, $B(E 2)$ value, PES.
\end{abstract}

\section{Introduction}

The low-lying states of the even-even ${ }^{178} \mathrm{Yb}^{186} \mathrm{Pt} \mathrm{nu}-$ clei could be described successfully in the framework of either phenomenological or microscopic models [12]. The Bohr- Mottelson Model (BMM) is significant for studying many of nuclei, where the vibrations are associated with mainly quadrupole oscillations of the nuclear surface. To study the rotational energy $E$ of some nuclei, a new relation of $I(I+1)$ has been introduced by Bohr and Mottelson [3] The bosonization of neutrons and protons in the shell model gives the interacting boson model (IBM). The interacting boson model involves two types of bosons called $s$ $(L=0)$ and $d(L=2)$, which play an important role in the reduction of the problems related to a nuclear structure. The interacting boson model contains three limiting symmetries, which are the rotational $\mathrm{SU}(3)$, vibrational $\mathrm{U}(5)$, and $\gamma$-unstable $\mathrm{O}(6)$ ones. In these limits, the nuclei may have transition properties, vibrational-rotational, vibrational- $\gamma$-unstable, and rotational- $\gamma$-unstable ones $[4,5]$. The interacting boson model (IBM-1) enables one to distinguish between the proton and neutron bosons. In the even-

(C) M. ABED AL-JUBBORI, 2017 even nuclei, the energy levels can be classified into the ground-state band (GSB) and $\beta$-band with $k^{\pi}=0^{+}$, as well as the $\gamma$-band with $k^{\pi}=2^{+}[6]$. Both bands, the ground-state and octupole ones can be described by the Interacting Vector Boson Model (IVBM), in which the bosons have collective excitation patterns in a nucleus. The IVBM is developed by Ganev et al. [7]. A new Semiempirical Formula (SEF) that depends on the angular momentum and energy levels is proposed by Al-Jubbori et al. [8]. This relation $(\mathrm{SEF})$ is able to calculate the rotational and vibrational energies of the even-even nuclei. In even-even nuclei, a single octupole band with levels characterized by $I^{\pi}=0^{+} 1^{-} 2^{+} 3^{-} 4^{+}, \ldots$ formed from the two bands, the GSB with $I^{\pi}=0^{+}, 2+4^{+}, \ldots$, and the negative-parity band (NPB) with $I^{\pi}=1^{-}, 3^{-}, 5^{-} \ldots$ [9-24]. This is an example of the odd-even staggering or $\Delta I=1$ staggering, the latter term is due to the fact that each energy level with angular momentum $I$ is displaced relatively to its neighbors with angular momenta $I= \pm 1[22]$. The aim of the present work is to study the low-excitation levels by the above mentioned models. In this study, the IBM-1is used in the calculations of energy levels for GSB, reduced transition probabilities $B(E 2)$ of ${ }^{178} \mathrm{Yb}^{186} \mathrm{Pt}$, and the po-

ISSN 2071-0194. Ukr. J. Phys. 2017. Vol. 62, No. 11 
tential energy surfaces. The NPB and GSB energy are calculated, by using IVBM and BMM for these nuclei.

\section{Method of Calculations}

The Hamiltonian in IBM-1 can be expressed as $[1,15$, $16]$

$$
\begin{aligned}
& H=\varepsilon_{s}\left(s^{\dagger} \cdot \tilde{s}\right)+\varepsilon_{d}\left(d^{\dagger} \cdot \tilde{d}\right)+ \\
& +\sum_{L=0,2,4} \frac{1}{2}(2 L+1)^{1 / 2} C_{L}\left[\left[d^{\dagger} \times d^{\dagger}\right]^{(L)} \times[\tilde{d} \times \tilde{d}]^{(L)}\right]^{(0)}+ \\
& +\frac{1}{\sqrt{2}} v_{2}\left[\left[d^{\dagger} \times d^{\dagger}\right]^{(2)} \times[\tilde{d} \times \tilde{s}]^{(2)}+\left[d^{\dagger} \times s^{\dagger}\right]^{(2)} \times\right. \\
& \left.\times[\tilde{d} \times \tilde{d}]^{(2)}\right]^{(0)}+\frac{1}{2} v_{0}\left[\left[d^{\dagger} \times d^{\dagger}\right]^{(0)}[\tilde{s} \times \tilde{s}]^{(0)}+\right. \\
& \left.+\left[s^{\dagger} \times s \dagger\right]^{(0)} \times[\tilde{d} \times \tilde{d}]^{(0)}\right]^{(0)}+\frac{1}{2} u_{0}\left[\left[s^{\dagger} \times s \dagger\right]^{(0)} \times\right. \\
& \left.\times[\tilde{s} \times \tilde{s}]^{(0)}\right]^{(0)}+u_{2}\left[\left[d^{\dagger} \times s \dagger\right]^{(2)} \times[\tilde{d} \times \tilde{s}]^{(2)}\right]^{(0)}
\end{aligned}
$$

There are two terms of Hamiltonian one-body interactions, $\left(\varepsilon_{s}\right.$ and $\left.\varepsilon_{d}\right)$, and seven terms of two-body interactions $\left[c_{L}(L=0,2,4), v_{L}(L=0,2), 0 u_{L}(L=0,2)\right]$, where $\varepsilon_{s}$ and $\varepsilon_{d}$ are the single-boson energies, and $c_{L}$, $v_{L}$ and $u_{L}$ describe the two-boson interactions. However, the $N$ pairs, $N=n_{s}+n_{d}$, represent the total number of bosons, and it is conserved [15]. Thus, the general relation (1) can be written as [16]

$\hat{H}=\varepsilon \hat{n}_{d}+a_{0} \hat{P} \cdot \hat{P}+a_{1} \hat{L} \cdot \hat{L}+a_{2} \hat{Q} \cdot \hat{Q}+a_{3} \hat{T}_{3} \cdot \hat{T}_{3}+$

$+a_{4} \hat{T}_{4} \cdot \hat{T}_{4}$

where $\hat{n}_{d}=\left(d^{\dagger} \cdot \tilde{d}\right)$ represents the boson energy operator, the pairing operator interaction is represented by $\hat{p}=1 / 2[(\tilde{d} \cdot \tilde{d})-(\tilde{s} \cdot \tilde{s})], \hat{L}=\sqrt{10}\left[d^{\dagger} \times \tilde{d}\right]^{-1}$ represents the third term of the relation, which is the contribution of the angular momentum $\mathrm{O}(3)$. The quadrupole interaction of the $L=2 d$-bosons is represented by the fourth term. The last two terms represent $\hat{T}_{r}=\left[d^{\dagger} \times \tilde{d}\right]^{r}$, where $(r=3)$ and $(r=4)$ are the octoupole and hexadecapole interaction operators, respectively.

The quadrupole operator is given by $[15,17]$

$\hat{Q}=\left[d^{\dagger} \times \tilde{S}+s^{\dagger} \times \tilde{d}\right]^{(2)}+\chi\left[d^{\dagger} \times \tilde{d}\right]^{(2)}$,

where $\chi$ is the quadrupole structure parameter and takes the values 0 and $\pm \frac{\sqrt{7}}{2}[15,17]$.

ISSN 2071-0194. Ukr. J. Phys. 2017. Vol. 62, No. 11
The eigenvalues for these three limits are given by [18]

$$
\begin{aligned}
& E=\varepsilon n d+\beta n d(n d+4)+2 \gamma v(v+3)+ \\
& +2 \delta L(L+1) \ldots U(5), \\
& E=\frac{a_{2}}{2}\left(\lambda^{2}+\mu^{2}+\lambda \mu+3(\lambda+\mu)\right)+ \\
& +\left(a_{1}-\frac{3 a_{2}}{8}\right) L(L+1) \ldots S U(3), \\
& E=a_{0} / 4(N-\sigma)(N+\sigma+4)+a_{3} / 2 \tau(\tau+3)+ \\
& +\left(a_{1}-a_{3} / 10\right) L(L+1) \ldots O(6),
\end{aligned}
$$

where $\beta, \gamma$, and $\delta$ represent the values of parameters.

The eigenvalues of energy levels for the groundstate band and negative party states in the interacting vector boson model are given by [20-24]

$E(I)=a I(I+1)+b I$,

$E(I)=a I(I+1)+(b+\eta) I+\zeta$,

where $a$ and $b$ can be estimated from the fit of the positive ground-state band, whereas the two parameters $\eta$ and $\zeta$ are estimated from the negative party state ones.

The energy levels of the ground and negative bands in $\mathrm{BMM}$ are given by $[3,16]$

$$
\begin{aligned}
& E(I)=A I(I+1)-B I^{2}(I+1)^{2}+C I^{3}(I+1)^{3}, \\
& E(I)=E_{0}+A^{\prime} I(I+1)-B^{\prime} I^{2}(I+1)^{2}+C^{\prime} I^{3}(I+1)^{3},
\end{aligned}
$$

where $E_{0}$ represents the band head energy of the negative party state, while the parameters $A^{\prime}, B^{\prime}$ and $C^{\prime}$ can be estimated from the fit of the available energy levels of NPB.

\section{Results and Discussion}

Based on the interacting boson model, the energy levels of the ground state, $\gamma, \beta$ bands, $B(E 2)$ values, and the potential energy surfaces are calculated. The interacting vector boson model and Bohr-Mottelson model were used to calculate the energy levels of the negative parity band. The results are discussed separately as follows.

\subsection{Energy levels}

The total boson numbers $N_{b}=N_{\pi}+N_{\nu}$, where, $N_{\pi}, N_{\nu}$ represent the bosons of a proton and a neutron respectively. Even-even nuclei have atomic numbers $Z=72$ to 80 , while the even neutron number 


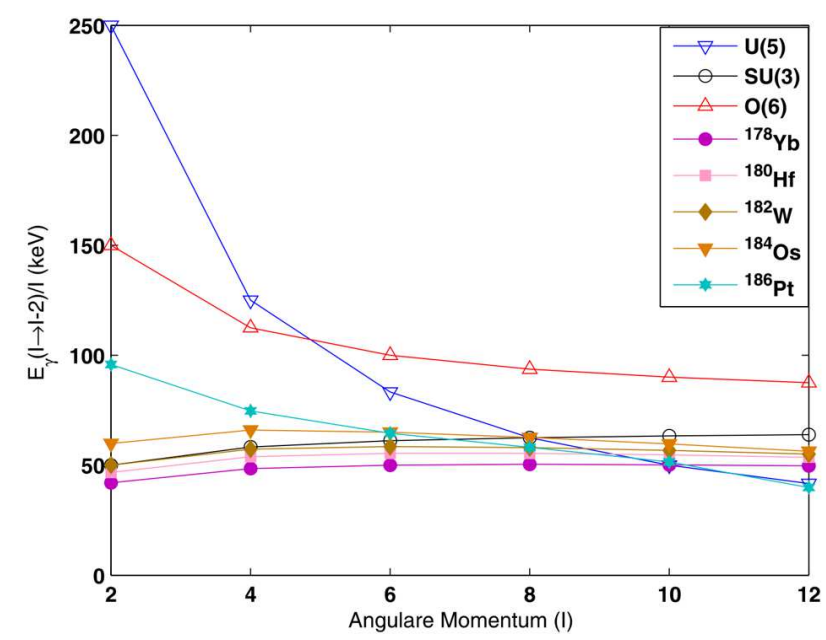

Fig. 1. (Color online) Energy gamma over spin of the groundstate band for ${ }^{178} \mathrm{Yb}-{ }^{186} \mathrm{Pt}$ nuclei [25-29]

for all ${ }^{178} \mathrm{Yb}^{186} \mathrm{Pt}$ nuclei is $N=108$. They have $(8-$ 2) proton pairs less than the magic number $Z=82$ and 9 neutron hole pairs less than the magic number $Z=126$. Therefore, the total boson numbers $N_{b}$ are 15 to 11 .

The ratio $R_{4 / 2}=E 4_{1}^{+} / E 2_{1}^{+}$is significant to distinguish the symmetry shape of a nucleus. It is $10 / 3$ for deformed nuclei, while 2.5 for $\gamma$-unstable nuclei and 2 for vibrational nuclei [1].

Table 1 shows the experimental values of $E=$ $=E 4_{1}^{+} / E 2_{1}^{+}$of these nuclei. In this table, $R_{4 / 2}$ attains the $\mathrm{SU}(3)$ value of $\sim 3.3$ for ${ }^{178} \mathrm{Yb}^{-184} \mathrm{Os} \mathrm{nu}-$ clei except ${ }^{186} \mathrm{Pt}$ with $R_{4 / 2}=2.5601$, which attains $\mathrm{O}(6)$. According to our analysis, ${ }^{178} \mathrm{Yb}^{-184}$ Os nuclei present features of SU(3)-nuclei, whereas ${ }^{186} \mathrm{Pt}$ nuclei present features of $\mathrm{O}(6)$-nuclei.

Regan et al. [30] introduced the relation $R=$ $=E \gamma(I \rightarrow I-2) / I$ energy gamma over spin (E-

Table 1. Experimental excitation energies (MeV) [25-29] for ${ }^{178} \mathrm{Yb}-{ }^{186} \mathrm{Pt}$

\begin{tabular}{|l|l|l|}
\hline \multirow{2}{*}{ Nuclei } & \multicolumn{2}{|c|}{ Experimental } \\
\cline { 2 - 3 } & $E\left(2_{1}^{+}\right)$ & $R=E\left(4_{1}^{+}\right) / E\left(2_{1}^{+}\right)$ \\
\hline $178 \mathrm{Yb}$ & 0.084000 & 3.3095 \\
$180 \mathrm{Hf}$ & 0.09332 & 3.3065 \\
${ }^{182} \mathrm{~W}$ & 0.100105 & 3.2908 \\
${ }^{184} \mathrm{Os}$ & 0.11977 & 3.2035 \\
$186 \mathrm{Pt}$ & 0.19153 & 2.5601 \\
\hline
\end{tabular}

GOS), this relation provides a valuable information about the evolution that appears in the yrast line of the even-even nuclei. For the three limits, these relations are given by [30]

$$
\begin{array}{ll}
\text { Vibrational } R=\frac{h \omega}{I} \rightarrow 0, & \text { when } I \rightarrow \infty, \\
\text { Rotational } R=\frac{\hbar^{2}}{2 \vartheta}\left(4-\frac{2}{I}\right) \rightarrow 4 \frac{\hbar^{2}}{2 \vartheta}, & \text { when } I \rightarrow \infty, \\
\gamma-\operatorname{soft}: R=\frac{E_{2_{1}^{+}}}{4}\left(1+\frac{2}{I}\right) \rightarrow \frac{E_{2_{1}^{+}}}{4} & \text { when } I \rightarrow \infty .
\end{array}
$$

From the above equations, the curve drops quickly from the highest value $(\approx 250 \mathrm{keV})$ at $\left(I=2_{1}^{+}\right)$ and equals to $(0)$ at $(I \rightarrow \infty)$ for vibrational nuclei. For the $\gamma$-soft nuclei, the situation is different, since the highest value $(\approx 150 \mathrm{keV})$ at $\left(I=2_{1}^{+}\right)$, and the curve drops gradually to $\frac{E_{2_{1}^{+}}}{4}$ at $(I \rightarrow \infty)$. The curve for rotational nuclei increases slowly from the smallest value $(\approx 50 \mathrm{keV})$ at $\left(I=2_{1}^{+}\right)$to $4 \frac{\hbar^{2}}{2 \vartheta}$ at $(I \rightarrow \infty)$ [30].

Figure 1 shows a comparison between the ideal limits mentioned earlier and the experimental curves for these nuclei. From this figure, the ${ }^{178} \mathrm{Yb}^{184} \mathrm{Os}$ possess the $\mathrm{SU}(3)$ limit, while the ${ }^{186} \mathrm{Pt}$ has the $\gamma$-soft limit.

The systematics of the energy ratios $r\left(\frac{I+2}{I}\right)$ of successive levels of collective bands in medium and heavy mass even-even nuclei was studied [31, 32].

The ratios for the given band of each spin $I$ were built to define the symmetry of the excited band of even-even nuclei in $[31,32]$ :

$r\left(\frac{I+2}{I}\right)=\left[R\left(\frac{I+2}{I}\right)_{\exp }-\frac{I+2}{I}\right] \times \frac{I(I+1)}{I(I+2)}$,

$R\left(\frac{I+2}{I}\right)_{\exp }$ represents the experimental energy ratio between the $I+2$ and $I$ states. The ratios $r((I+2) / I)$ with $I=2,4,6, \ldots$ have been studied as well. These ratios show distinctly different behaviors in the vibrational, rotational, and $\gamma$-unstable limits. The ratio $r$ should be close to 0 and to 1 for vibrational and rotational nuclei, respectively. While, it should have values spanning between zero and one for $\gamma$ unstable nuclei. In Eq. (10), the value of energy ratios $(r)$ changes between 0.1 and 1 for GSB of even-even

ISSN 2071-0194. Ukr. J. Phys. 2017. Vol. 62, No. 11 
nuclei [31, 32]:

$$
\left.\begin{array}{ll}
0.1 \leq r \leq 0.35 & \text { for vibrational nuclei, } \\
0.4 \leq r \leq 0.6 & \text { for transitional nuclei, } \\
0.6 \leq r \leq 1.0 & \text { for rotational nuclei. }
\end{array}\right]
$$

Figure 2 shows the ratio $r\left(\frac{I+2}{I}\right)$ as a function of $I$ for the GSB of ${ }^{178} \mathrm{Yb}-{ }^{186} \mathrm{Pt}$ nuclei. The plot is used to distinguish between different kinds of collective behavior of rotational $\mathrm{SU}(3)$, vibrational $\mathrm{U}(5)$, and $\gamma$-unstable $\mathrm{O}(6)$ nuclei. From this figure, the ratios $r((I+2) / I)$ start with a value very close to one and then constantly decrease with $I$ to $\leqslant 0.6$ for ${ }^{178} \mathrm{Yb}-$ ${ }^{184} \mathrm{Os}$, so this confirms that the nuclei have a rotational limit. While, the curve of ${ }^{186} \mathrm{Pt}$ is close to 0.4 and 0.6 , which confirms that ${ }^{186} \mathrm{Pt}$ has $\mathrm{O}(6)$ limit.

The interacting boson model, interacting vector boson model, and Bohr-Mottelson model were used to calculated the energy levels of GSB, $\gamma, \beta$, and NPB with special MATLAB software and the PHINT code written by Scholten [32]. In Tables 2 and 3, the number of bosons together with the best values of the parameters [25-29] for ${ }^{178} \mathrm{Yb}-{ }^{186} \mathrm{Pt}$ nuclei are summarized.

The calculated GSB, $\beta$ - and $\gamma$-bands and experimental data $[25-29]$ for ${ }^{178} \mathrm{Yb}^{186} \mathrm{Pt}$ nuclei are shown in Fig. 3, where the results of calculations are in good agreement with the experiment for these nuclei.

Levels with "( )" in GBS, $\beta$ and $\gamma$-band correspond to the cases where the spin and/or parity of the corresponding states are not well established experimentally.

Table 4 shows the results of calculations, which are performed within IBVM and BMM and are reliable to predict the negative party band for all nuclei. This table shows that the BMM calculations are in a good agreement with the experimental data for these bands and better than those of IVBM, except ${ }^{178} \mathrm{Yb}$ nuclei, since there is no sufficient experimental data for the NPB band. Levels with "*" correspond to the cases where the spin and/or parity of the corresponding states are not well established experimentally.

The odd-even staggering can be calculated by the equation [33]

$$
\begin{aligned}
& \Delta E_{1, \gamma}(I)=1 / 16\left[6 E_{1, \gamma}(I)-4 E_{1, \gamma}(I-1)-\right. \\
& \left.-4 E_{1, \gamma}(I+1)+E_{1, \gamma}(I-2)+E_{1, \gamma}(I+2)\right],
\end{aligned}
$$

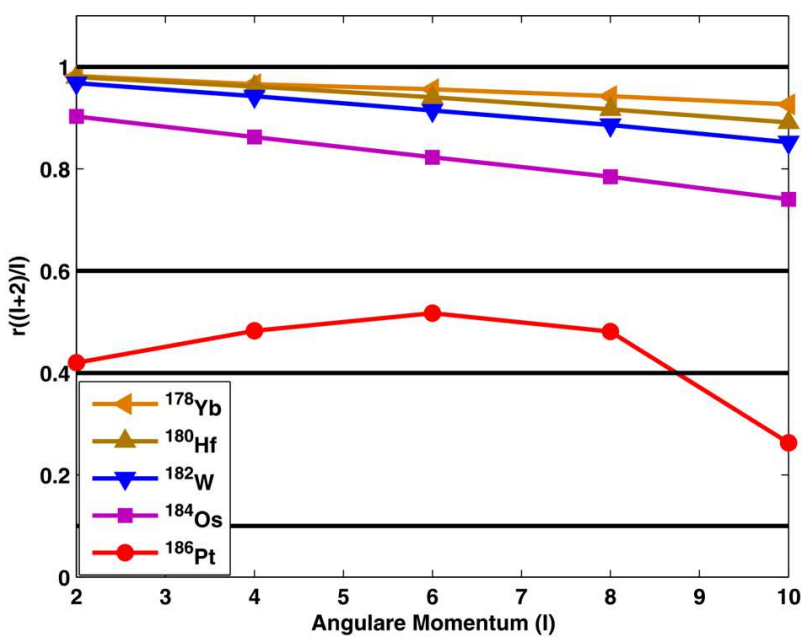

Fig. 2. (Color online) Ratio versus $I$ for ${ }^{178} \mathrm{Yb}^{-186} \mathrm{Pt}$ nuclei [25-29]

where $E_{1, \gamma}(I)=E_{1, \gamma}(I+1)-E(I) \Delta E_{1, \gamma}(I)$ exhibits the values of alternating sign over the extended region of the angular momentum. In general, the staggering starts from relatively high values and then gradually decreases, as $I$ increases. Following that, the staggering starts to raise and then drops again. The phase change appears when the staggering reaches a vanishing value $[9,19]$. The odd-even staggering results are shown in Fig. 4 for ${ }^{180} \mathrm{Hf}-{ }^{186} \mathrm{Pt}$ nuclei. From this figure, the IVBM and BMM results slightly decrease with increasing $I$, and it is in good agreement with experimental data. The staggering curves do not reach zero, which confirms that ${ }^{180} \mathrm{Hf}-{ }^{184} \mathrm{Os}$ possess $\mathrm{SU}(3)$ properties, whereas the ${ }^{186} \mathrm{Pt}$ has $\mathrm{O}(6)$.

\section{4. $B(E 2)$ Values}

The electrical transition can be also calculated under the framework of IBM, and the most general E2 transition operator can be written as $[1,8,18]$

$T^{E 2}=\alpha_{2}\left[d^{\dagger} s+s^{\dagger} d\right]^{(2)}+\beta_{2}\left[d^{\dagger} d\right]^{(2)}=e_{B} \hat{Q}$,

where $\left(s^{\dagger}, d^{\dagger}\right)$ and $(s, d)$ represent the creation and annihilation operators for $s$ and $d$ bosons, respectively, while $\alpha_{2}$ and $\beta_{2}$ are two parameters, where $\beta_{2}=\chi \alpha_{2}, \alpha_{2}=e_{B}$ are the effective charge of a boson and the quadrupole operator $Q$. The matrix elements of the $T^{E 2}$ operator can give the reduced transition rates as [8, 34-35]

$B\left((E 2) l_{i} \rightarrow L_{f}\right)=\frac{1}{2 L_{i}+1}\left|\left\langle L_{f}|| T^{(E 2)}|| L_{i}\right\rangle\right|^{2}$. 

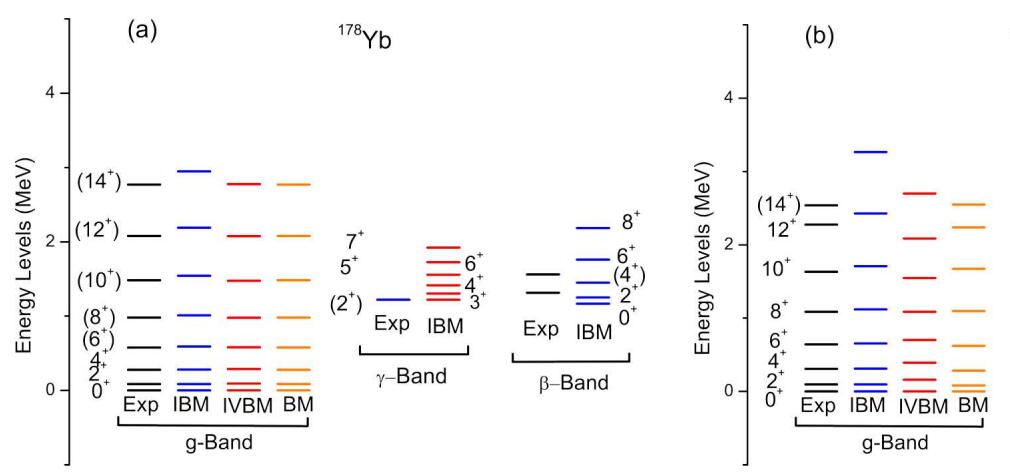

${ }^{180} \mathrm{Hf}$
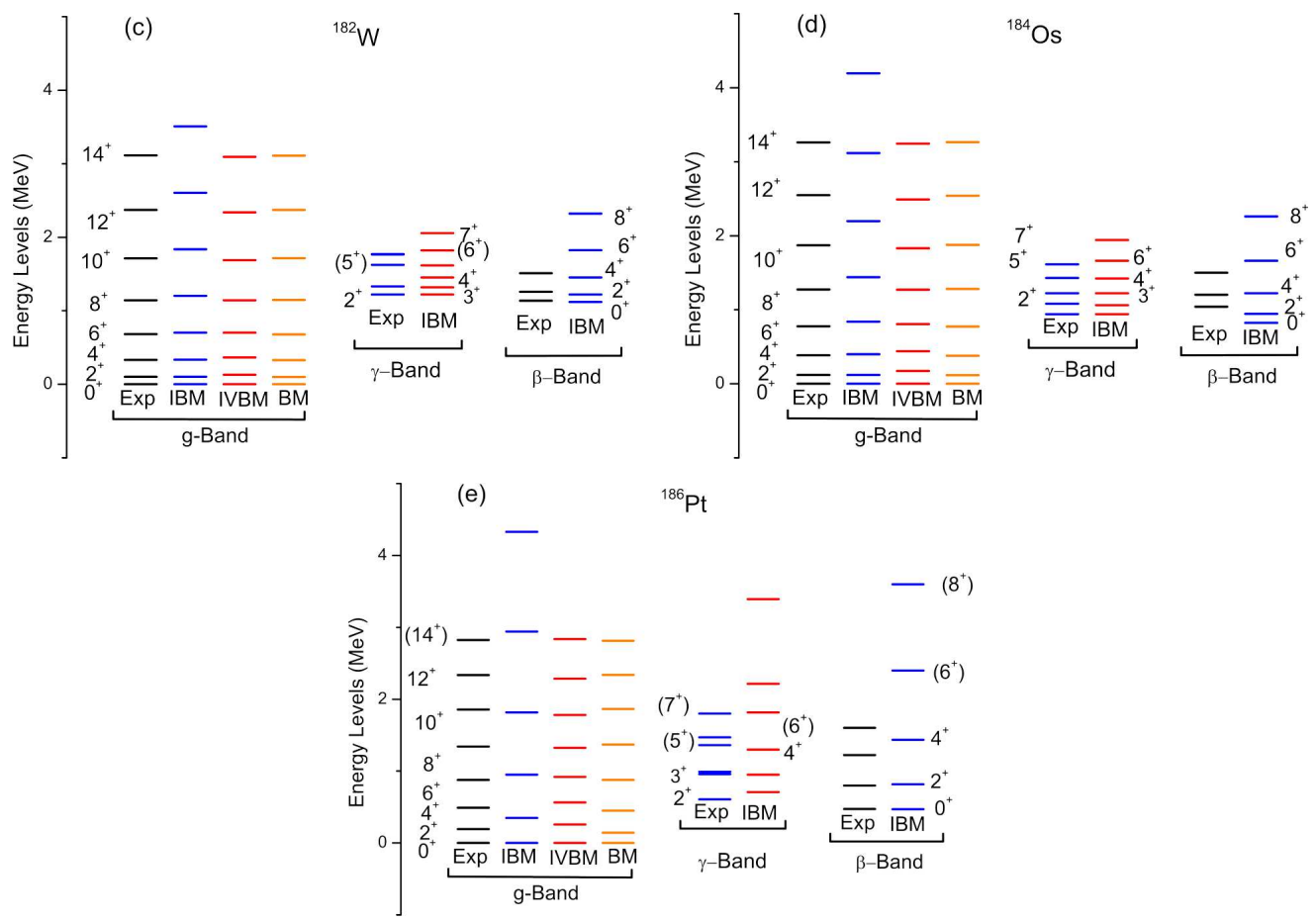

${ }^{186} \mathrm{Pt}$
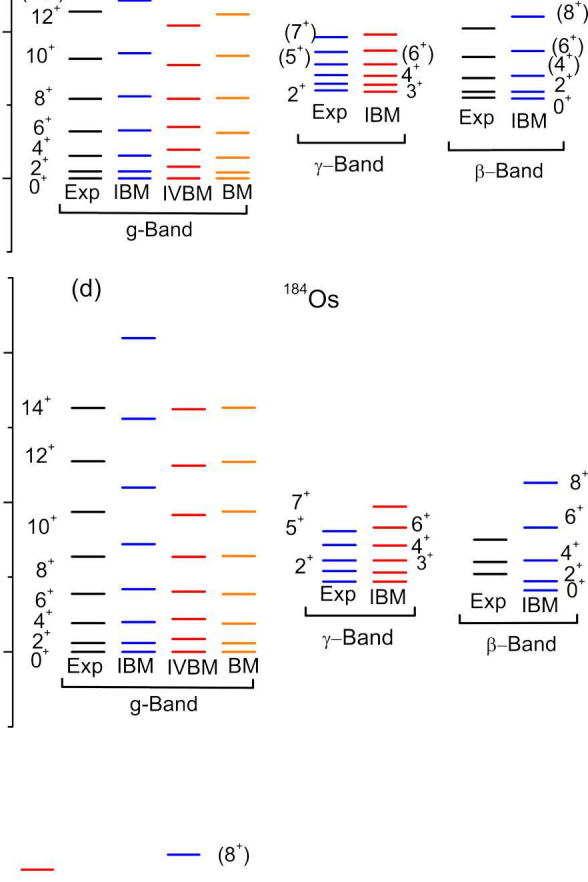

$\underset{\operatorname{Exp}}{\equiv 3^{+}} \equiv 0^{+}$

$\gamma$-Band $\underset{\beta \text {-Band }}{\longleftarrow}$

IVBM, and BMM for ${ }^{178} \mathrm{Yb}-{ }^{186} \mathrm{Pt}$ nuclei

Table 2. Parameters in MeV of IBM-1,

IVBM, and BMM used in the calculation of ${ }^{178} \mathrm{Yb}-{ }^{186} \mathrm{Pt}$ nuclei

\begin{tabular}{|c|c|c|c|c|c|c|r|r|r|r|c|}
\hline \multirow{2}{*}{ Nuclei } & \multirow{2}{*}{$N_{b}$} & \multicolumn{5}{|c|}{ IBM } & \multicolumn{3}{c|}{ IVBM } & \multicolumn{3}{c|}{ BM } \\
\cline { 3 - 11 } & & QQ & ELL & PAIR & OCT & CHI & $a \times 10^{-3}$ & $b \times 10^{-3}$ & $A$ & $B \times 10^{-3}$ & $C \times 10^{-3}$ \\
\hline${ }^{178} \mathrm{Yb}$ & 15 & -0.0261 & 0.0182 & - & - & -1.333 & 12.7013 & 7.8545 & 13.972 & 5.1982 & 0.7027 \\
$180 \mathrm{Hf}$ & 14 & -0.0269 & 0.0210 & - & - & -1.333 & 9.4928 & 50.3726 & 13.511 & -0.3952 & -21.956 \\
$182 \mathrm{w}$ & 13 & -0.0299 & 0.0222 & - & - & -1.333 & 12.9957 & 25.9124 & 16.533 & 9.1374 & 0.4463 \\
$184 \mathrm{Os}$ & 12 & -0.0239 & 0.0310 & - & - & -1.333 & 12.1950 & 48.9146 & 19.336 & 0.2373 & 2.6987 \\
$186 \mathrm{Pt}$ & 11 & - & 0.0589 & 0.0196 & 0.0242 & 0.000 & 6.1512 & 110.3425 & 23.877 & 0.7739 & 13.073 \\
\hline
\end{tabular}

$\left(\mathrm{PAIR}=a_{0} / 2, \mathrm{ELL}=2 a_{1}, \mathrm{QQ}=2 a_{2}, \mathrm{OCT}=a_{3} / 5\right)[18]$. 

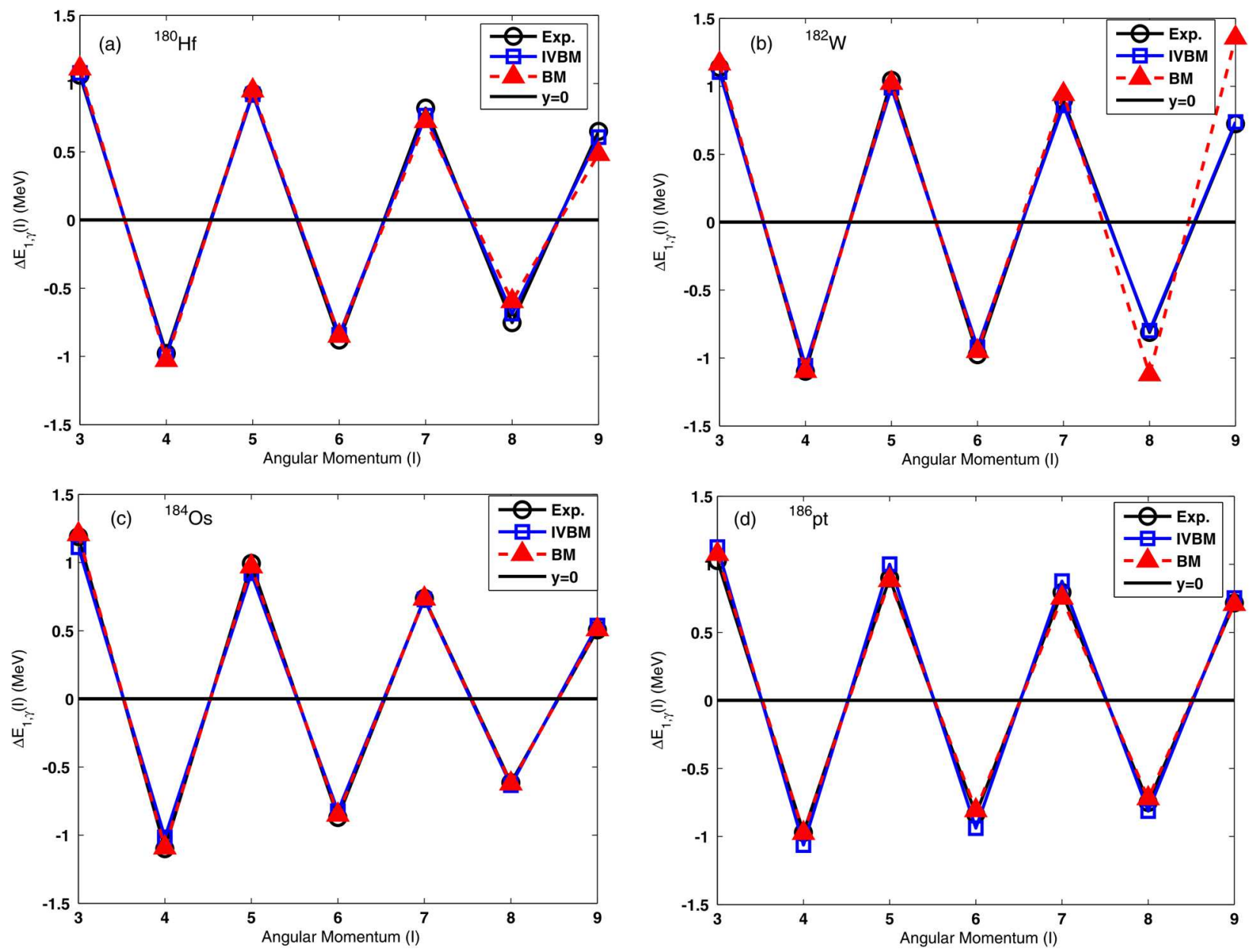

Fig. 4. (Color online) Staggering calculated from Eq. (12) for ${ }^{180} \mathrm{Hf}-{ }^{186} \mathrm{Pt}$ nuclei

${ }^{180} \mathrm{Hf}-{ }^{184}$ Os nuclei are an excellent example to study the behavior of the total low-lying $E 2$ strengths. The effective charge, $\alpha_{2}=e_{B}$, can be determined from the experimental $B(E 2) ; 2_{1}^{+} \rightarrow 0_{1}^{+}$. Table 5 shows the values of the $\alpha_{2}$ and $\beta_{2}$ parameters. The electromagnetic transition rates for the experimental values and the IBM calculation are listed in Table 6 for ${ }^{180} \mathrm{Hf}-{ }^{184} \mathrm{Os}$ nuclei.

\subsection{Potential energy surface}

These three symmetry limits form a triangle known as the Casten triangle and represent the nuclear phase diagram [18], and all states in IBM-1 possess positive parity, while the octupole effects within the interacting boson framework can be described in the framework of the spdf-IBM, introduced by Engel and ISSN 2071-0194. Ukr. J. Phys. 2017. Vol. 62, No. 11
Table 3. IVBM and BMM parameters of NPB in MeV used in the calculation of ${ }^{180} \mathrm{Hf}-{ }^{186} \mathrm{Pt}$ nuclei

\begin{tabular}{|c|c|c|c|c|c|}
\hline \multirow{2}{*}{ Nuclei } & \multicolumn{2}{|c|}{ IVBM } & \multicolumn{3}{c|}{ BM } \\
\cline { 2 - 6 } & \multicolumn{1}{|c|}{$\zeta$} & $\eta$ & $A \times 10^{-3}$ & $B \times 10^{-3}$ & $C \times 10^{-3}$ \\
& & & & & \\
\hline${ }^{180} \mathrm{Hf}$ & 1.4290 & -0.0783 & -2.9235 & -18.787 & -7.4972 \\
${ }^{182} \mathrm{~W}$ & 1.37383 & -0.0606 & 7.0609 & -4.4987 & -1.5444 \\
${ }^{184} \mathrm{Os}$ & 1.5437 & -0.0958 & 4.1831 & -5.5743 & -1.7726 \\
${ }^{186} \mathrm{Pt}$ & 1.4077 & -0.0625 & 7.6315 & -4.5248 & -1.6648 \\
\hline
\end{tabular}

Iachello [34, 35]. At that time, Kusnezov and Iachello gave a detailed study of the ${ }^{140-148} \mathrm{Ba}$ isotopes [36] and then the spdf-IBM was developed by Kusnezov and Zamfir [39-42]. 
Table 4. Experimental and calculated energy levels in MeV within IVBM and BMM of NPB for ${ }^{180} \mathrm{Hf}-{ }^{186} \mathrm{Pt}$

\begin{tabular}{|c|c|c|c|c|c|c|}
\hline \multirow{2}{*}{$I$} & \multicolumn{3}{|c|}{${ }^{180} \mathrm{Hf}$} & \multicolumn{3}{|c|}{${ }^{182} \mathrm{~W}$} \\
\hline & Exp. & IVBM & $\mathrm{BM}$ & Exp. & IVBM & $\mathrm{BM}$ \\
\hline 3 & $1.4298^{*}$ & 1.4600 & 1.4205 & 1.37383 & 1.3738 & 1.4648 \\
\hline 5 & $1.444^{*}$ & 1.5750 & 1.4910 & 1.62128 & 1.6213 & 1.6220 \\
\hline 7 & $1.7651^{*}$ & 1.7660 & 1.7236 & 1.91705 & 1.8588 & 1.8832 \\
\hline 9 & $2.1342^{*}$ & 2.0330 & 2.1419 & 2.27387 & 2.2313 & 2.2611 \\
\hline 11 & $2.588^{*}$ & 2.3759 & 2.5931 & 2.71093 & 2.7077 & 3.7345 \\
\hline 13 & $2.603^{*}$ & 2.7947 & 2.6011 & 3.22453 & 3.2881 & 3.2180 \\
\hline \multirow{2}{*}{$I$} & \multicolumn{3}{|c|}{${ }^{184} \mathrm{Os}$} & \multicolumn{3}{|c|}{${ }^{186} \mathrm{Pt}$} \\
\hline & Exp. & IVBM & BM & Exp. & IVBM & BM \\
\hline 3 & 1.5437 & 1.5493 & 1.6016 & 1.40771 & 1.6249 & 1.5055 \\
\hline 5 & 1.71807 & 1.6750 & 1.7146 & $1.69273^{*}$ & 1.8312 & 1.6729 \\
\hline 7 & $1.95843^{*}$ & 1.8983 & 1.9216 & 1.95233 & 2.0868 & 1.9477 \\
\hline 9 & $2.22183^{*}$ & 2.2191 & 2.2425 & $2.37492^{*}$ & 2.3915 & 2.3379 \\
\hline 11 & $2.6615^{*}$ & 2.6375 & 2.6595 & $2.788^{*}$ & 2.7459 & 2.8026 \\
\hline 13 & $3.0835^{*}$ & 3.1539 & 3.0829 & $3.2998^{*}$ & 3.1486 & 3.2918 \\
\hline
\end{tabular}

Table 5. Parameters (in eb) used to reproduce $B(E 2)$ values for ${ }^{180} \mathrm{Hf}-{ }^{186} \mathrm{Pt}$ nuclei

\begin{tabular}{|c|c|c|c|}
\hline Isotope & $N_{b}$ & $\alpha_{2}$ & $\beta_{2}$ \\
\hline${ }^{180} \mathrm{Hf}$ & 14 & 0.1037 & -0.1371 \\
${ }^{182} \mathrm{~W}$ & 13 & 0.1055 & -0.1355 \\
${ }^{184} \mathrm{Os}$ & 12 & 0.0998 & -0.1320 \\
${ }^{186} \mathrm{Pt}$ & 11 & 0.1320 & 0.0000 \\
\hline
\end{tabular}

IBM was formulated initially in terms of the creation and annihilation boson operators. Its geometric interpretation was given in terms of shape variables by introducing the intrinsic coherent state, which is expressed as a boson condensate [8, 36]:

$|N, \beta, \gamma\rangle=1 / \sqrt{N !}\left(b_{c}^{\dagger}\right)^{N}|0\rangle$,

where $|0\rangle$ denotes the boson vacuum, and

$b_{c}^{\dagger}=\left(1+\beta^{2}\right)^{-1 / 2}\left\{s^{\dagger}+\beta\left[\cos \gamma\left(d_{0}^{\dagger}\right)+\right.\right.$

$\left.\left.+\sqrt{1 / 2} \sin \gamma\left(d_{2}^{\dagger}+d_{-2}^{\dagger}\right)\right]\right\}$,

where $N$ is the boson number, $\beta$ measures the total deformation of a nucleus, while $\gamma$ measures a deviation from the axial symmetry, which determines the geometrical shape of the nucleus.

Here, $\beta \geqslant 0$ and $0<\gamma<\pi / 3, \beta$ and $\gamma$ have been given in [8].
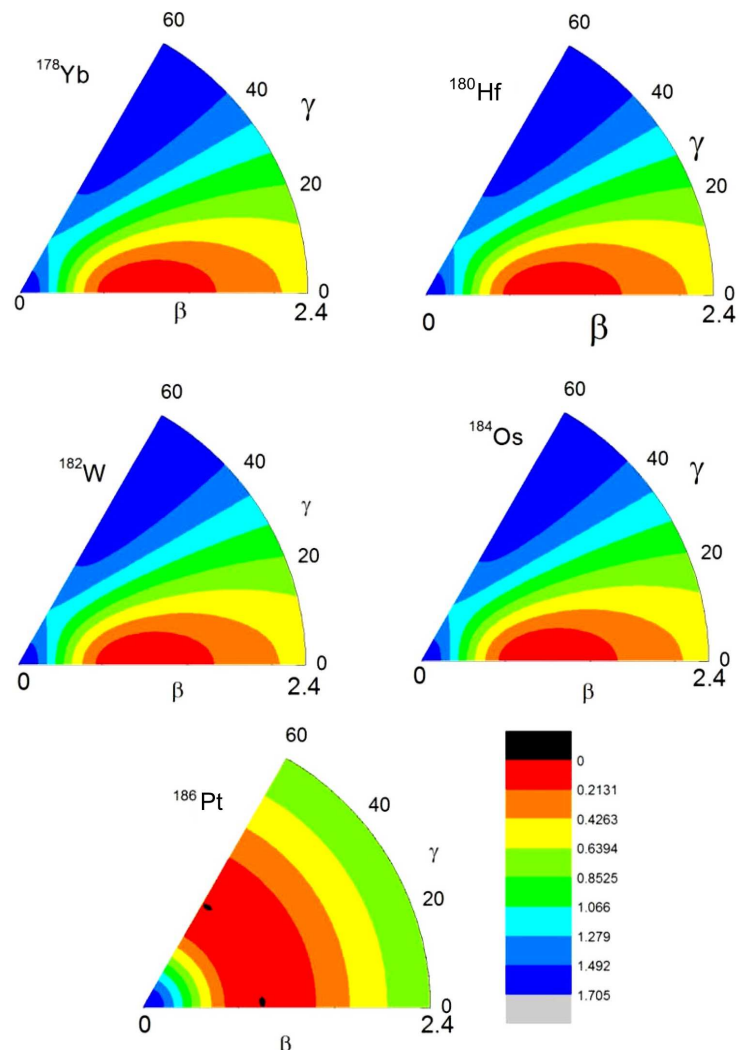

Fig. 5. (Color online) Potential energy surfaces for ${ }^{178} \mathrm{Yb}-$ ${ }^{186} \mathrm{Pt}$ nuclei

ISSN 2071-0194. Ukr. J. Phys. 2017. Vol. 62, No. 11 
Table 6. IBM-1 and the experimental data [25-29] of $B(E 2)$ (in $\left.e^{2} b^{2}\right)$ for ${ }^{180} \mathbf{H f}-{ }^{186} \mathrm{Pt}$ nuclei

\begin{tabular}{|c|c|c|c|c|c|c|c|c|}
\hline \multirow{2}{*}{$\begin{array}{l}\text { Isotopes } \\
J_{i} \rightarrow J_{f}\end{array}$} & \multicolumn{2}{|c|}{${ }^{180} \mathrm{Hf}$} & \multicolumn{2}{|c|}{${ }^{182} \mathrm{~W}$} & \multicolumn{2}{|c|}{${ }^{184} \mathrm{Os}$} & \multicolumn{2}{|c|}{${ }^{186} \mathrm{Pt}$} \\
\hline & Exp. & IBM-1 & Exp. & IBM-1 & Exp. & IBM-1 & Exp. & IBM-1 \\
\hline $2_{1}^{+} \rightarrow 0_{1}^{+}$ & 0.935 & 0.931 & 0.833 & 0.837 & 0.619 & 0.644 & 0.593 & 0.757 \\
\hline $4_{1}^{+} \rightarrow 2_{1}^{+}$ & 1.389 & 1.315 & 1.201 & 1.180 & 0.870 & 0.906 & & 0.796 \\
\hline $2_{2}^{+} \rightarrow 0_{1}^{+}$ & & & 0.0208 & 0.00 & & 0.000 & & 0.079 \\
\hline $5_{1}^{+} \rightarrow 4_{2}^{+}$ & & 0.708 & & 0.624 & & 0.467 & & 0.021 \\
\hline $6_{1}^{+} \rightarrow 4_{1}^{+}$ & 1.322 & 1.418 & 1.232 & 1.269 & $0.0027<$ & 0.969 & & 0.888 \\
\hline $6_{2}^{+} \rightarrow 4_{2}^{+}$ & & 0.852 & & 0.749 & & 0.561 & & 0.609 \\
\hline $6_{2}^{+} \rightarrow 5_{1}^{+}$ & & 0.527 & & 0.466 & & 0.351 & & 0.000 \\
\hline $6_{3}^{+} \rightarrow 4_{3}^{+}$ & & 1.125 & & & & 0.734 & & 0.622 \\
\hline $8_{1}^{+} \rightarrow 6_{1}^{+}$ & 1.479 & 1.436 & 1.281 & 1.281 & $0.008<$ & 0.972 & & 0.912 \\
\hline $8_{2}^{+} \rightarrow 6_{2}^{+}$ & & 0.994 & & 0.869 & & 0.646 & & 0.608 \\
\hline $10_{1}^{+} \rightarrow 8_{1}^{+}$ & 1.437 & 1.416 & 1.244 & 1.251 & $0.0033<$ & 0.941 & & 0.891 \\
\hline $12_{1}^{+} \rightarrow 10_{1}^{+}$ & & 1.364 & 1.170 & 1.193 & $0.0018<$ & 0.886 & 0.044 & 0.836 \\
\hline $14_{1}^{+} \rightarrow 12_{1}^{+}$ & & 1.287 & 1.042 & 1.111 & & 0.811 & & 0.753 \\
\hline
\end{tabular}

From Hamiltonian (1), the potential energy surfaces (PES) were calculated from the intrinsic boson condensate state (14):

$$
\begin{aligned}
& E\left(N_{b}, \beta, \gamma\right)= \\
& =\left\langle N_{b}, \beta, \gamma|H| N_{b}, \beta, \gamma\right\rangle /\left\langle N_{b}, \beta, \gamma \mid N_{b}, \beta, \gamma\right\rangle= \\
& =\frac{N_{b} \varepsilon_{d} \beta^{2}}{\left(1+\beta^{2}\right)}+\frac{N_{b}\left(N_{b}+1\right)}{\left(1+\beta^{2}\right)^{2}} \times \\
& \times\left(\alpha_{1} \beta^{4}+\alpha_{2} \beta^{3} \cos 3 \gamma+\alpha_{3} \beta^{2}+\alpha_{4}\right) .
\end{aligned}
$$

These expressions give, for large $N_{b}, \beta_{\min }=0,1.414$, and 1 for $\mathrm{U}(5), \mathrm{SU}(3)$, and $\mathrm{O}(6)$, respectively. The calculated potential energy surfaces are shown in Fig. 5. It can be seen from the figure that the eveneven ${ }^{178} \mathrm{Yb},{ }^{180} \mathrm{Hf},{ }^{182} \mathrm{~W}$, and ${ }^{184} \mathrm{Os}$ nuclei under study are deformed and have rotational symmetry $\mathrm{SU}(3)$, except ${ }^{186} \mathrm{Pt}$ which has $\mathrm{O}(6)$.

\section{Conclusions}

In conclusion, the energy levels are calculated, by using IBM-1, IVBM and BMM for ${ }^{178} \mathrm{Yb}^{-186} \mathrm{Pt}$ nuclei with $A=178$ to 186 . The analysis shows a good agreement of the results of these models and the available experimental data. The energy gamma over spin curves of the GSB for ${ }^{178} \mathrm{Yb}-{ }^{186} \mathrm{Pt}$ nuclei are plotted and compared with the ideal limits of vibrational, rotational and $\gamma$-soft cases. From this study, ${ }^{178} \mathrm{Yb}-$ ${ }^{184}$ Os nuclei have the rotational property, while the
${ }^{186} \mathrm{Pt}$ has the $\mathrm{O}(6)$ property. The ratio $r\left(\frac{I+2}{I}\right)$ has been applied to describe the GSB of the above nuclei. The study has demonstrated that the ground and octupole bands exhibit $\Delta I=1$ staggering, and the vanishing value of the staggering $\Delta E 1, \gamma(I)=0$ has not been reached. The reduced transition probabilities $B(E 2)$ of these nuclei are calculated,by using IBM-1. The potential energy surfaces have confirmed that ${ }^{178} \mathrm{Yb}-{ }^{184} \mathrm{Os}$ nuclei possess $\mathrm{SU}(3)$, and ${ }^{86} \mathrm{Pt}$ has $\mathrm{O}(6)$ characteristics.

The author thanks University of Mosul, College of Education for Pure Science, Department of Physics for supporting this work.

1. F. Iachello, A. Arima, The Interacting Boson Model, (Cambridge Univ. Press, 1987).

2. F. Iachello. Analytic description of critical point nuclei in a spherical-axially deformed shape phase transition. Phys. Rev. Lett. 87, 052502 (2001).

3. A. Bohr, B.R. Mottelson. Nuclear Structure, Vol. II (Word Scientific, 1998).

4. P. Cejnar, J. Jolie, R.F. Casten. Quantum phase transitions in the shapes of atomic nuclei. Rev. Mod. Phys. 82, 2155 (2010).

5. R.F. Casten, E.A. McCutchan. Quantum phase transitions and structural evolution in nuclei. J. Phys. G 34, R285 (2007).

6. F. Iachello. Dynamic symmetries at the critical point. Phys. Rev. Lett. 85, 3580 (2000).

7. H. Ganev, V.P. Garistov, A.I. Georgieva. Description of the ground and octupole bands in the symplectic extension of the interacting vector boson model. Phys. Rev. C 69, 014305 (2004). 
8. M.A. Al-Jubbori, H.H. Kassim, F.I. Sharrad, I. Hossain. Nuclear structure of even ${ }^{120-136} \mathrm{Ba}$ under the framework of IBM, IVBM and new method (SEF). Nucl. Phys. A 955, 101 (2016).

9. I. Mamdouh, M. Al-Jubbori. The rotational-vibrational properties of ${ }^{178-188}$ Os isotopes. Indian J. Phys. 89, 1085 (2015).

10. S.N. Abood, M.A. Al-Jubbori. Nuclear structure and electromagnetic transitions investigation in Er isotopes within framework of interacting boson model. Commun. Theor. Phys. 60, 335 (2013).

11. E.H. Hagemann. Electromagnetic transitions from levels in the beta-vibrational band of ${ }^{174}$ Hf. Nucl. Phys. A 161, 449 (1971).

12. T. Morikawa et al. Coulomb excitation of ${ }^{174} \mathrm{Hf} \mathrm{K}$-isomer. $\mathrm{X}$-ray spectroscopy with high-spin isomer beam. Phys. Lett. B 350, 169 (1995).

13. K. Nomura et al. Microscopic formulation of the interacting boson model for rotational nuclei. Phys. Rev. C 83, 041302 (2011).

14. H.H. Kassim, A.A. Mohammed-Ali, F.I. Sharrad, I. Hossain, K. S. Jassim. Nuclear structure of even ${ }^{178-182} \mathrm{Hf}$ isotopes under the framework of interacting boson model (IBM-1). Iran. Jou. Sci. Technol. Trans. Sci. DOI: 10.1007/s40995-016-0104-x.

15. A. Arima, F. Iachello. Interacting boson model of collective nuclear states II. The rotational limit. Ann. Phys. N.Y. 111, 201 (1978).

16. N.V. Zamfir, D. Kusnezov. Octupole correlations in the transitional actinides and the spdf interacting boson model. Phys. Rev. C 63, 054306 (2001).

17. F. Iachello. Dynamical supersymmetries in nuclei. Phys. Rev. Lett. 44, 772 (1980).

18. R.F. Casten, D.D. Warner. The interacting boson approximation. Rev. Mod. Phys. 60, 389 (1988).

19. A. Georgieva, P. Raychev, R. Roussev. Interacting twovector-boson model of collective motions in nuclei. J. Phys. G: Nucl. Phys. 8, 1377 (1982).

20. B. Singh. Nuclear data sheets for $A=172$. Nuclear Data Sheets 75, 199 (1995).

21. A. Georgieva, P. Raychev, R. Roussev. Rotational limit of the interacting two-vector boson model. J. Phys. G: Nucl. Phys. 9, 521 (1983).

22. A. Georgieva, P. Raychev, R. Roussev. Interacting vector boson model and negative parity bands in actinides. Bulg. J. Phys. 12, 147 (1985).

23. P. Raychev. Unified description of positive and negative parity states in the deformed heavy nuclei by means of two interacting vector bosons. Rev. Roum. Phys. 32, 471 (1987).

24. E. Acheterbeg, O.A. Capurro, G.V. Marti. Nuclear data sheets for $A=178$. Nucl. Data Sheets 110, 1473 (2009).

25. E.A. McCutchan. Nuclear data sheets for $A=180$. Nucl. Data Sheets 126, 151 (2015).

26. B. Singh. Nuclear data sheets for $A=182$. Nucl. Data Sheets 130, 21 (2015).

27. C.M. Baglin. Nuclear data sheets for $A=184$. Nucl. Data Sheets 111, 275 (2009).
28. P.H. Regan et al. Signature for vibrational to rotational evolution along the yrast line. Phys. Rev. Lett. 90, 152502 (2003).

29. D. Bonatsos, L.D Skouras. Successive energy ratios in medium- and heavy-mass nuclei as indicators of different kinds of collectivity. Phys. Rev. C 43, 952R (1991).

30. O. Scholten. Computer code PHINT, KVI (Groningen, 1980).

31. D. Bonatsos et al. $\Delta I=1$ staggering in octupole bands of light actinides: "Beat" patterns. Phys. Rev. C 62, 024301 (2000).

32. M.A. Al-Juboori, K. Al-Mtiuty, Kh.I. Saeed, F.I. Sharrad. Properties of even ${ }^{168-178} \mathrm{Hf}$ isotopes using IBM-1 and SEF. Chin. Phys. C 41 (8) 084103 (2017).

33. H.H. Kassim, F.I. Sharrad. Energy levels and electromagnetic transition of ${ }^{190-196} \mathrm{Pt}$ nuclei. Inter. J. of Mod. Phys. E 23, 1450070 (2014).

34. J. Engel, F. Iachello. Quantization of asymmetric shapes in nuclei. Phys. Rev. Lett. 54, 1126 (1985).

35. J. Engel, F. Iachello. Interacting boson model of collective octupole states: (I). The rotational limit. Nucl. Phys. A 472, 61(1987).

36. D. Kusnezov, F. Iachello. A study of collective octupole states in barium in the interacting boson model. Phys. Lett. B 209, 420 (1988).

37. D. Kusnezov. The U(16) algebraic lattice. J. Phys. A: Math. Gen. 22, 4271 (1989).

38. D. Kusnezov. The U(16) algebraic lattice. II. Analytic construction. J. Phys. A: Math. Gen. 23, 5673(1990).

39. N. V. Zamfir, D. Kusnezov. Octupole correlations in the transitional actinides and the spdf interacting boson model. Phys. Rev. C 63, 054306 (2001).

40. N.V. Zamfir, D. Kusnezov. Octupole correlations in U and Pu nuclei. Phys. Rev. C 67, 014305 (2003).

41. A.E.L. Dieperink, O. Scholten, F. Iachello. Classical limit of the interacting-boson model. Phys. Rev. Lett. 44, 1747 (1980).

Received 06.10.17

М. Абед Ал-Джсабборі

\section{ВИВЧЕННЯ РІВНІВ ЕНЕРГЇ̈}

I ЕЛЕКТРОМАГНІТНИХ ПЕРЕХОДІВ У $\mathrm{Yb}-\mathrm{Pt}$

ЯДРАХ $3 N=108$ У ВБ, ВВБ, І БМ МОДЕЛЕЙ

$\mathrm{P}$ е $з$ ю м е

У моделях взаємодіючих бозонів (МВБ), взаємодіючих векторних бозонів (МВВБ) і Бора-Моттельсона (МБМ) описані рівні енергії і електромагнітні переходи в ядрах ${ }^{178} \mathrm{Yb}$ ${ }^{186} \mathrm{Pt}(N=108)$. Для визначення еволюції основного стану розраховані відношення $r((I+2) / I)$ і $R=E \gamma(I \rightarrow I-2) / I$ як функції спіна $I$. У МВВБ і МБМ розраховані смуги 3 негативною парністю і смуга в основному стані, тоді як у МВБ розраховані $R, \gamma, \beta$ і приведені ймовірності переходів $B(E 2)$ у хорошій згоді з експериментальними даними. Для власного когерентного стану визначені поверхні потенціальної енергії. 3 цих результатів випливає, що обертання цих ядер характеризується $\mathrm{SU}(3)$ симетрією, a ${ }^{186} \mathrm{Pt} \mathrm{O}(6)$ симетрією.

ISSN 2071-0194. Ukr. J. Phys. 2017. Vol. 62, No. 11 